\title{
Electroweak bremsstrahlung and indirect detection of Dark Matter by neutrino telescopes
}

\author{
N. Baro ${ }^{\star a}$, M. Beneke ${ }^{a}$, M. Krämer ${ }^{a}$, M. Rummel ${ }^{a, b}$ \\ a Institut für Theoretische Teilchenphysik und Kosmologie, \\ D-52056 Aachen, Germany \\ ${ }^{b}$ Institut für Theoretische Physik \\ D-22761 Hamburg, Germany
}

Electroweak bremsstrahlung in supersymmetry relevant in the context of indirect detection of dark matter through neutrino detection is analyzed. We discuss scenarios with $\mathrm{TeV}$ dark matter in the MSSM where electroweak bremsstrahlung enhancements become important for neutrino flux detection. In these scenarios the dark matter particle is a pure bino neutralino and slepton masses are close to the LSP. We present our results at the creation point and also for neutrino telescope prospects.

Identification of Dark Matter 2010

July 26 - 302010

University of Montpellier 2, Montpellier, France

*Speaker. 


\section{Introduction}

The nature of dark matter is one of the most notable unsolved problems in particle physics and there have been many attempts to identify its nature via direct or indirect detection experiments. Prospects are promising for the future due to continually improving experimental sensitivity. Furthermore, collider experiments like the LHC might discover candidates for the dark matter particle, for example by discovering the lightest supersymmetric particle in the near future. In this paper, we will discuss electroweak bremsstrahlung (EWBS) in dark matter annihilation. EWBS stands for the radiation of electroweak gauge bosons $Z$ and $W^{ \pm}$in an annihilation process. For energies above the electroweak scale, EWBS of $W$ or $Z$ bosons might induce important corrections due to Sudakov logarithms[1-6]. In the following we will focus on possible enhancement resulting of EWBS to neutrino flux coming from annihilation of dark matter. Indirect detection of dark matter through neutrino flux observation can become promising searches with the help of neutrino telescopes such as the IceCube experiment. It would be then interesting to argue whether such an enhancement can play a role on the future detection of dark matter. Indirect neutrino flux predictions in three-body annihilation of dark matter have been done in Ref. [7] for gaugino neutralino annihilating into $t \bar{b} W$. In EWBS processes, neutrinos can be produced primarily in the annihilation hard process and in the decay of the radiated gauge bosons which also lead to other secondaries like electrons, positrons, gamma-rays and anti-protons that are interesting messenger particles for indirect detection. We present the expected enhanced neutrino flux on earth within the MSSM resulting of electroweak bremsstrahlung processes at high neutralino mass. This paper is organized as follows. In section 2 , we present an overview of our calculation. In section 3 we analyze electroweak bremsstrahlung effects in the MSSM and we introduce a scenario where EWBS is important for neutrino signals. We then discuss the resulting neutrino flux at creation in section 4 and the neutrino signal on earth from annihilation in the sun in section 5. Finally we summarize and conclude in section 6 .

\section{Overview of the calculation}

The observed flux of neutrinos on earth $d \Phi_{v} / d E_{v}$ is proportional to the spectrum of neutrinos per annihilation $d N_{v}^{f} / d E_{v}$ at production,

$$
\frac{d \Phi_{v}}{d E_{v}}=\Gamma \sum_{f} B_{f} \frac{d N_{v}^{f}}{d E_{v}},
$$

where $B_{f}$ stands for the branching ratio of dark matter annihilation to a partial final state giving neutrinos and $\Gamma$ is related to the astrophysical context of neutrino production. For instance, in the case of neutrino production in the sun, $\Gamma$ is the annihilation rate given by

$$
\Gamma=\frac{C_{a}}{2} N^{2}(t)=\frac{C_{c}}{2} \tanh ^{2}\left(\frac{t}{\tau}\right), \quad \tau=\frac{1}{\sqrt{C_{c} C_{a}}},
$$

where $N(t)$ is the total number of neutralinos being trapped at a time $t, C_{c}$ is the capture rate and $C_{a}$ is the annihilation cross-section times the relative dark matter velocity per volume. When the equilibrium is reached the annihilation rate depends only on the capture rate $C_{c}$, however this is not automatically fulfilled in all possible MSSM parameter points, especially the one considered in the 
following where co-annihilation of dark matter is important to the relic density[8]. We consider in addition to the standard annihilation channels $f \bar{f}, W^{+} W^{-}, Z Z$ and $H^{0} A^{0}, Z h^{0}, Z H^{0}, W^{ \pm} H^{ \pm}$threebody final state processes $\tilde{\chi}_{1}^{0} \tilde{\chi}_{1}^{0} \rightarrow v \bar{v} Z, v e^{+} W^{-}$. First we present the enhancement produced by EWBS at the creation point in section 4. MadGraph[9] is used to generate SLHA event files for the two-to-three hard process. We use the SLHA interface of PYTHIA[10] to read in the files produced by MadGraph and let the primarily produced $Z, W^{ \pm}$and $\tau^{ \pm}$decay. Second we would like to see the impact of EWBS corrections to neutrino production resulting from dark matter annihilation in the sun, see section 5. In order to obtain the detectable neutrino flux which can be seen on earth, we need to insert the neutrino spectra produced with MadGraph and PYTHIA into WimpSim[11] to propagate the neutrinos to the earth, i.e. to 1 Astronomical Unit (AU). For annihilations in the sun the muons $\mu^{ \pm}$, pions $\pi^{ \pm}$, kaons $K^{ \pm}$and the neutron $n$ are made stable since they interact with the material in the sun and are absorbed before they can decay and produce neutrinos. An interface between the flux produced at creation and WimpSim has been created. If we have three particles in the final state of the hard process the cms energy is continuously spread over those three particles and their energy distribution might depend on the specific choice of the supersymmetric model. Hence, if we want to study the contribution of EWBS processes to the neutrino flux we cannot just simulate the decay of the hard process final states like in WimpSim but need to also simulate the hard process itself. Our modified WimpSim performs the remaining steps to propagate the neutrinos to the earth and result tables are read with DarkSUSY[12] to calculate the neutrino flux at IceCube.

\section{Electroweak bremsstrahlung in the MSSM}

The MSSM does not allow any primary production of neutrinos from two-body final state annihilations of dark matter. This is due to the so-called helicity suppression occured in annihilation of neutralinos into a pair of light leptons. Indeed the matrix element of the amplitude is proportional to the mass of the leptons in the final state. For neutrinos, such processes are then completely forbidden. It is then interesting to notice that primary production of neutrinos is restored in supersymmetry in electroweak bremsstrahlung processes such as $\tilde{\chi}_{1}^{0} \tilde{\chi}_{1}^{0} \rightarrow v \bar{v} Z, v e^{+} W^{-}$(where $v$ and $e$ stand for any lepton generation). The Feynman diagrams for the $Z$-bremsstrahlung are shown in Fig. 1. Note that the $W$-bremsstrahlung has an analogue diagrammatic structure.

First, one should remember that we are considering annihilation at the zero velocity. It follows that the two s-channel diagrams 1.b) and 1.c) are completely suppressed in this limit. Second, one remarks that the two Initial State Radiation diagrams 1.a) and 1.d) are dominant for a higgsino- or wino-like neutralino whereas the Intermediate State Radiation diagram 1.e) and Final State Radiation diagram 1.f) are dominant for a bino-like neutralino but are suppressed for heavy sneutrinos. In addition one can clearly see that diagrams 1.a) and 1.b) are mostly included in Monte Carlo simulations as subprocesses of annihilation of dark matter into gauge bosons $\tilde{\chi}_{1}^{0} \tilde{\chi}_{1}^{0} \rightarrow Z Z / W^{+} W^{-}$ where one of the two gauge bosons decays on-shell into a pair of neutrinos. In such scenarios, the three-body final state annihilation processes are already mostly included as a by-product of the two-body gauge boson final state ones. Finally we expect large enhancement from electroweak bremsstrahlung processes for a heavy neutralino mass due to Sudakov logarithms. Therefore we have chosen a model demanding an heavy neutralino mass in order to get an important electroweak 


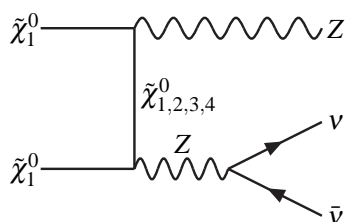

a)

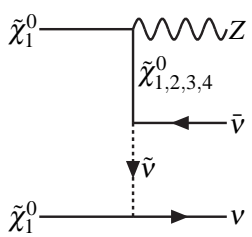

d)

Initial State Radiation

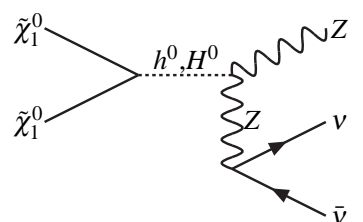

b)

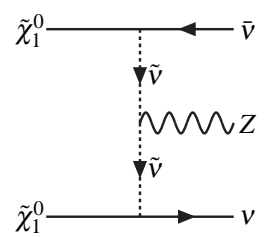

e)

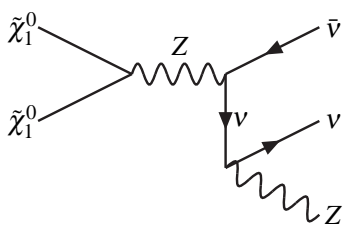

c)

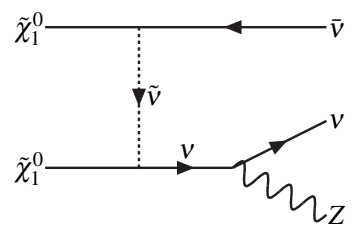

f)

Figure 1: Generic Feynman diagrams for $\tilde{\chi}_{1}^{0} \tilde{\chi}_{1}^{0} \rightarrow v \bar{v} Z$.

bremsstrahlung effect, a pure bino-like neutralino with a sneutrino mass close to the neutralino one to focus on contributions coming from 1.e) and 1.f) and also taking a relic density which is compatible with the actual experimental bounds. This is checked by using DarkSUSY[12]. For illustration we choose the following set of parameters. The chargino/neutralino system is fixed by taking $M_{1}=3 \mathrm{TeV}, M_{2}=9 \mathrm{TeV}, \mu=9 \mathrm{TeV}$ in order to get a bino neutralino of about 3.1 $\mathrm{TeV}$. The slepton masses are about $m_{\tilde{l}}=3.1 \mathrm{TeV}$ whereas the squark masses are set to $9 \mathrm{TeV}$. For completeness we give the parameters in the Higgs sector: $\tan \beta=10$ and $M_{A^{0}}=9 \mathrm{TeV}$.

For small relative velocity, the total two-body annihilation cross sections contributes to $\sigma v^{2 \rightarrow 2}=$ $2.86 \times 10^{-6} \mathrm{pb}$ whereas the total $2 \rightarrow 3$ contributions give $\sigma v^{2 \rightarrow 3}=3.59 \times 10^{-6} \mathrm{pb}$ representing a large correction of about $126 \%$ compared to the standard contribution. The relic density calculated from all $2 \rightarrow 2$ cross sections with coannihilation with DarkSUSY[12] is $\Omega h^{2}=0.064$.

\section{Neutrino flux at creation}

Even though we treat all the individual channels separately, Fig. 2 shows the sum of all the flavor and neutrino/antineutrino contributions for simplicity. Note that the spectrum for the electron/muon neutrinos and for tau neutrinos are different but we show the overall contribution only. As we are working in the zero velocity limit and as we are interested in models where the slepton mass is close to the neutralino mass, one can get a simple approximated formula for the spectrum of neutrino $d N_{v} / d z$ resulting of the process $\tilde{\chi}_{1}^{0} \tilde{\chi}_{1}^{0} \rightarrow v \bar{v} Z$,

$$
\begin{gathered}
\frac{d N_{v}}{d z} \simeq \frac{1}{2 z^{2}}\left[-2 z^{3}-2 z^{2} \log (1-z)+5 z^{2}+8 z \log (1-z)-8 z-8 \log (1-z)\right] \\
+\frac{\varepsilon}{(z-1) z^{3}}\left[z^{5}-11 z^{3}+4 z^{3} \log (1-z)-18 z^{2} \log (1-z)+19 z^{2}\right. \\
+22 z \log (1-z)-8 z-8 \log (1-z)]
\end{gathered}
$$

where $z=E_{v} / m_{\chi_{1}^{0}}$ and $\varepsilon=\left(m_{\tilde{v}}-m_{\chi_{1}^{0}}\right) / m_{\chi_{1}^{0}}$. Note that the relation is valid for a pure bino neutralino. The formula (4.1) does not take into account the decay of $Z$ boson which increases the neutrino flux at light energies namely $z \lesssim 0.1$. One can observe in Fig. 2 that the three-body final 


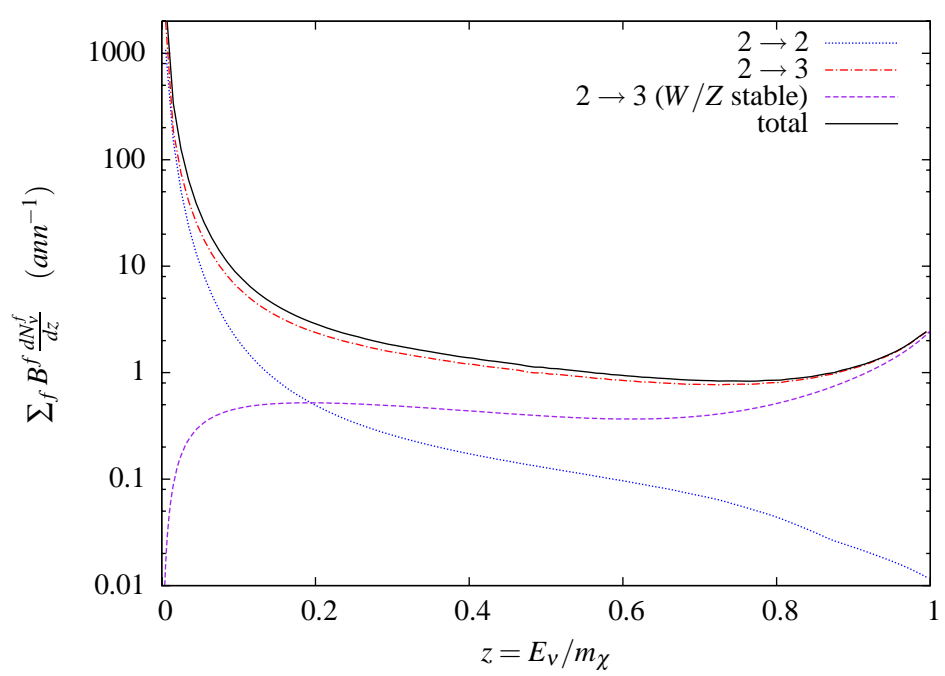

Figure 2: Total neutrino spectrum at creation. The blue dotted curve represents the sum of all the standard $2 \rightarrow 2$ contributions whereas the red dot-dashed curve represents the sum of all the $2 \rightarrow 3$ ones: $\tilde{\chi}_{1}^{0} \tilde{\chi}_{1}^{0} \rightarrow$ $v \bar{v} Z, v e^{+} W^{-}$. The purple dashed curve represents the $2 \rightarrow 3$ spectrum with stable gauge bosons. The black straight curve shows the total.

state processes give a very large enhancement especially at high energies. Such EWBS corrections should be then included in expected neutrino flux. The neutrino spectrum obtained in Fig. 2 have to be multiplied by an astrophysical factor $\Gamma$ in Eq. (2.1). In the next section we present the case of the annihilation at the core of the sun and the resulting neutrino signals detected on earth by IceCube.

\section{Neutrino flux from the sun}
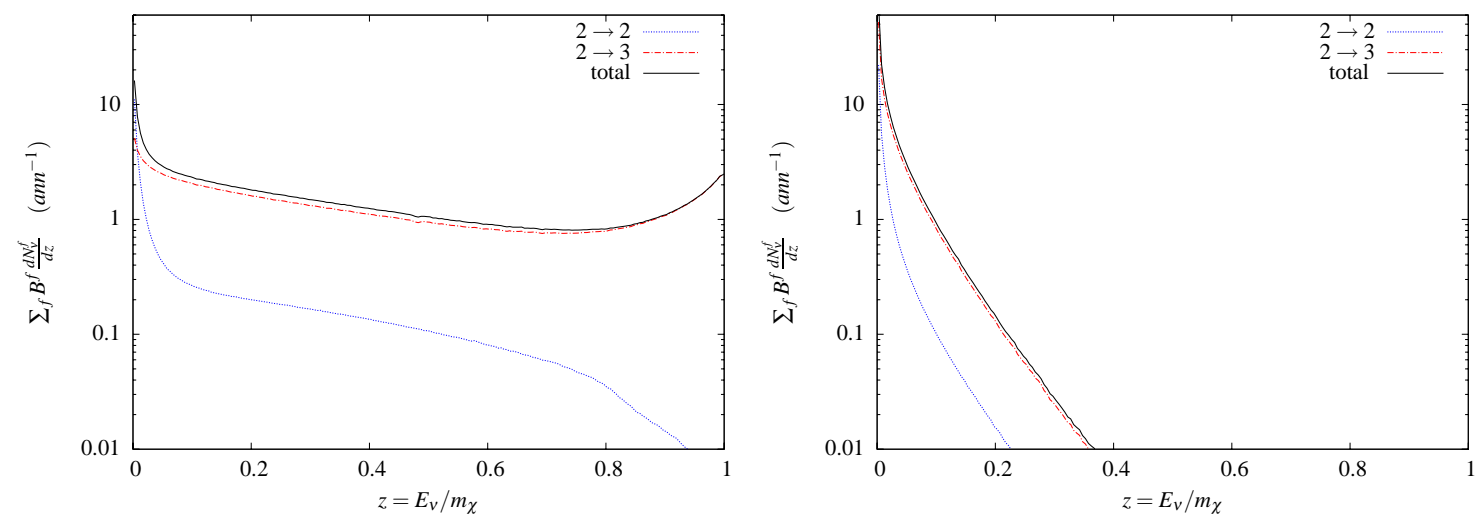

Figure 3: Total neutrino spectrum at the center (left panel) and at the surface of the sun (right panel).

We present our results for the neutrino flux $d N_{v} / d z$ at the center and at the surface of the sun in Fig. 3. Equation (4.1) tells us that the spectrum $d N_{v} / d z(z)$ of a generic $2 \rightarrow 3$ process does not depend explicitely on the neutralino mass when $m_{\tilde{v}} \sim m_{\tilde{\chi}}$. As all of these channels have a same 
branching ratio, the sum $\sum_{f} B_{f} \frac{d N_{v}^{f}}{d E_{v}}$ for models with different values of the neutralino mass have then the same shape. We clearly see in Fig. 3 that the high energy part of the neutrino spectrum gets absorbed in the sun. This is because high energy neutrinos $E_{v} \gtrsim 1 \mathrm{TeV}$ undergo many interactions in the sun and are absorbed as is discussed in Ref. [13] and Ref. [14, 15]. During the propagation in the sun, high energy neutrinos are suppressed by an exponential factor $e^{-z\left(m_{\tilde{\chi}} / \bar{\varepsilon}\right) r} . \bar{\varepsilon} \simeq 100 \mathrm{GeV}$ for neutrinos and $\bar{\varepsilon} \simeq 140 \mathrm{GeV}$ for antineutrinos and $r$ stands for a rescaled distance, $r=0$ at production and $r=1$ at the surface of the sun. For example, with a $3 \mathrm{TeV}$ dark matter mass, the flux gets a reduction of $95 \%$ (88\%) for neutrinos (antineutrinos) at $z=0.1$. This is a drawback for MSSM models with large $m_{\tilde{\chi}}$ since it seems that most of the neutrino flux from neutralino annihilation gets absorbed in the sun. In addition to the previous set of parameters called bino3000 in the following, we introduce two other benchmark models bino500 and bino500' to reduce the exponential suppression effect. In these two scenarios, we choose $\mu=1700 \mathrm{GeV}, M_{1}=500 \mathrm{GeV}$ and $M_{2}=1800 \mathrm{GeV}$. The slepton masses are about $540 \mathrm{GeV}$. In bino500 the squark masses are chosen to be very large whereas in bino500' we decrease the squark masses to $650 \mathrm{GeV}$. We then calculate the integrated flux of muon neutrinos and anti-muon neutrinos $\Phi_{v_{\mu}}$ per $\mathrm{km}^{2}$ and per year at IceCube for the $2 \rightarrow 2$ and the $2 \rightarrow 3$ annihilation processes. The results for our benchmark models bino3000, bino500 and bino500' are in Table 1.

\begin{tabular}{l|c|c} 
& $\Phi_{v_{\mu}}^{2 \rightarrow 2}$ & $\Phi_{v_{\mu}}^{2 \rightarrow 2+2 \rightarrow 3}$ \\
\hline bino3000 & $1.376 \cdot 10^{-4}$ & $6.987 \cdot 10^{-4}(80 \%)$ \\
bino500 & $5.337 \cdot 10^{3}$ & $6.104 \cdot 10^{+3}(12.6 \%)$ \\
bino500' & $6.713 \cdot 10^{6}$ & $6.808 \cdot 10^{+6}(1.4 \%)$
\end{tabular}

Table 1: Muon-neutrino fluxes $\left(v_{\mu}+\bar{v}_{\mu}\right)$ from the sun in $\mathrm{km}^{-2} \mathrm{yr}^{-1}$ at IceCube.

First of all, the ratio of the fluxes calculated in the last column of Table 1 tells us that EWBS processes can indeed have an impact on the total neutrino flux at IceCube, i.e. in models bino3000 and bino500 but not so much in bino500'. However, the flux varies over many orders of magnitude which cannot be explained just by the variation of $\sigma v$.

\begin{tabular}{c|c|c|c} 
& $\sigma_{p \chi}^{\mathrm{SI}}$ & $\sigma_{p \chi}^{\mathrm{SD}}$ & $\Gamma$ \\
\hline bino3000 & $1.37 \cdot 10^{-12}$ & $9.82 \cdot 10^{-13}$ & $7.2 \cdot 10^{-10}$ \\
bino500 & $2.44 \cdot 10^{-11}$ & $1.69 \cdot 10^{-8}$ & $3.2 \cdot 10^{-3}$ \\
bino500, & $9.13 \cdot 10^{-11}$ & $3.83 \cdot 10^{-7}$ & $5.64 \cdot 10^{0}$
\end{tabular}

Table 2: Cross sections $\sigma_{p \chi}^{\mathrm{SI}}, \sigma_{p \chi}^{\mathrm{SD}}$ in pb. Annihilation rate $\Gamma$ in $10^{24} \mathrm{yr}^{-1}$.

We see in Table 2 that the variation of $\Phi_{v_{\mu}}$ in our three benchmark models is mostly determined by the variation of the annihilation rate $\Gamma$. Clearly the influence of the spin-independent cross sections $\sigma_{p \chi}^{\mathrm{SI}}$ and the spin-dependent ones $\sigma_{p \chi}^{\mathrm{SD}}$ on $\Gamma$ is visible. In fact, increasing $\sigma_{p \chi}^{\mathrm{SI}}$ and $\sigma_{p \chi}^{\mathrm{SD}}$ was the motivation for lowering $m_{\tilde{q}}$ in the introduction of bino500' since lower squark masses increase these cross sections, which in turn increases the annihilation rate. As a result, we observe the largest muon neutrino flux for bino500' but also EWBS does not have a big significance in this scenario because the fraction of the corresponding flux is only $\sim 1 \%$. We note that our obtained values for 
the inelastic neutralino-proton scattering cross sections are below current limits of direct detection, $\sigma_{p \chi}^{\mathrm{SI}} \lesssim 10^{-7} \mathrm{pb}$ from the CDMS[16] and XENON[17] experiments and the less stringent limit on spin-dependent cross sections $\sigma_{p \chi}^{\mathrm{SD}} \lesssim 1 \mathrm{pb}$ from the COUPP[18] and KIMS[19] collaborations. Qualitatively the current limits on the neutrino induced muon-flux $\Phi_{\mu}$ at IceCube from Ref. [20] is around $10^{2} \mathrm{~km}^{-2} y r^{-1}$ for the three benchmark models we analyzed. However the expected order of magnitude calculated from Tab. 1 is about $10^{-12} \mathrm{~km}^{-2} y r^{-1}$ for bino3000, $10^{-5} \mathrm{~km}^{-2} y r^{-1}$ for bino500 and $10^{-2} \mathrm{~km}^{-2} y r^{-1}$ for bino500'. Comparing these results shows us that there is certainly no hope of detecting a scenario like bino3000 at IceCube. Also, the overall flux from bino500 is still seven orders of magnitude lower than the current limit of Ref. [20]. Even scenario bino500', where EWBS does not play a relevant role as we showed in Tab. 1 is four orders of magnitude away from current detection limits.

\section{Conclusion}

We have shown that EWBS can correct the predicted neutrino flux for a neutrino telescope in significant amounts. However the class of scenarios where this is possible produce an overall neutrino flux at the earth that may be too low to be detected with IceCube. The following features are responsible for these scenarios not to be detectable with IceCube. First, $\sigma v^{2 \rightarrow 2}$ has to be fairly small to obtain a significant relative contribution from EWBS processes which are limited to $\sigma v^{2 \rightarrow 3} \leq 10^{-4} \mathrm{pb}$ for scenarios where EWBS is not indirectly included in the two-body final state Monte Carlo simulation via the on-shell decays of gauge bosons. The small overall $\sigma v$ results in a rather small annihilation rate that is in turn crucial for the overall neutrino flux. Secondly, the analyzis of our models showed that for $\mathrm{TeV}$ neutralino the biggest part of high energy neutrinos is absorbed during the passage through the sun as shown and the contribution of EWBS processes to the overall $\sigma v$ is more important for these scenarios than for scenarios with neutralino mass below the $\mathrm{TeV}$ range. This drawback is of course absent for neutralino annihilations produced outside the sun, for example in the galactic halo. Thirdly, demanding the neutralino to be highly bino to suppress the $2 \rightarrow 2$ gauge boson channels $Z Z$ and $W W$ results in rather low scattering cross sections $\sigma_{p \chi}^{\mathrm{SI}}$ and $\sigma_{p \chi}^{\mathrm{SD}}$ since the $Z$ boson exchange channels are suppressed in the neutralino-proton scattering process. These results in a low capture rate of neutralinos in the sun which in turn results in a low annihilation rate.

A future prospect would be to analyze three-gauge boson final states, $W^{+} W^{-} Z$ and $Z Z Z$. In that case, neutrinos are produced secondarily and of course from subsequent decays. Such processes might lead to important corrections in expected neutrino signals. However some additional complications come into play due to radiative corrections and electroweak Coulomb-Sommerfeld effects.

\section{Acknowledgments}

This work is supported in part by the European Community's Marie-Curie Research Training Network under contract MRTN-CT-2006-035505 "Tools and Precision Calculations for Physics Discoveries at Colliders", the DFG SFB/TR9 "Computational Particle Physics", and the Helmholtz Alliance "Physics at the Terascale". 


\section{References}

[1] J. F. Beacom, N. F. Bell, G. D. Mack, Phys. Rev. Lett. 99 (2007) 231301, astro-ph/0608090.

[2] M. Kachelriess, P. D. Serpico, Phys. Rev. D76 (2007) 063516, 0707.0209 [hep-ph].

[3] N. F. Bell, J. B. Dent, T. D. Jacques, T. J. Weiler, Phys. Rev. D78 (2008) 083540, 0805.3423 [hep-ph].

[4] M. Kachelriess, P. D. Serpico, M. A. Solberg, Phys. Rev. D80 (2009) 123533, 0911.0001 [hep-ph].

[5] N. F. Bell, J. B. Dent, T. D. Jacques, T. J. Weiler, 1009.2584 [hep-ph].

[6] P. Ciafaloni, D. Comelli, A. Riotto, F. Sala, A. Strumia and A. Urbano, 1009.0224 [hep-ph].

[7] X. Chen, M. Kamionkowski, JHEP 9807 (1998) 001, hep-ph/9805383.

[8] J. Ellis, K. A. Olive, C. Savage, V. C. Spanos, 0912.3137 [hep-ph].

[9] F. Maltoni, T. Stelzer, hep-ph/0208156.

[10] T. Sjostrand, S. Mrenna, P. Z. Skands, Comput. Phys. Commun. 178 (2008) 852-867, hep-ph/0710.3820.

[11] M. Blennow, J. Edsjö, T. Ohlsson, 0709.3898 [hep-ph];

J. Edsjö, WimpSim Neutrino Monte Carlo, http://www.physto.se/ edsjo/wimpsim/.

[12] P. Gondolo et al., JCAP 0407 (2004) 008, astro-ph/0406204; http: //www.physto.se/ edsjo/darksusy/.

[13] M. Cirelli, N. Fornengo, T. Montaruli, I. Sokalski, A. Strumia, F. Vissani, Nucl. Phys. B727 (2005) 99, [Erratum-ibid. B790 (2008) 338], hep-ph/0506298.

[14] I. F. M. Albuquerque, L. Hui, E. W. Kolb, Phys. Rev. D64 (2001) 083504, hep-ph/0009017.

[15] P. Crotty, Phys. Rev. D66 (2002) 063504, hep-ph/0205116.

[16] Z. Ahmed et al. [CDMS Collaboration], Phys. Rev. Lett. 102 (2009) 011301, 0802.3530 [astro-ph].

[17] J. Angle et al. [XENON Collaboration], Phys. Rev. Lett. 100 (2008) 021303, 0706.0039 [astro-ph].

[18] E. Behnke et al. [COUPP Collaboration], Science 319 (2008) 933, 0804.2886 [astro-ph].

[19] H. S. Lee et al. [KIMS Collaboration], Phys. Rev. Lett. 99 (2007) 091301, 0704.0423 [astro-ph].

[20] J. Braun, D. Hubert and for the IceCube Collaboration, 0906.1615 [astro-ph.HE]. 